Batty, C., Berry, M., Dooley, K., Frankham, B., \& Kerrigan, S. (Eds.), The Palgrave Handbook of Screen Production, 2019, Palgrave Macmillan reproduced with permission of Palgrave Macmillan.

This extract is taken from the author's original manuscript and has not been edited. The definitive, published, version of record is available here: https://www.palgrave.com/gp/book/9783030217433 


\section{Mobile reception: Materiality and locality with small screens}

Bettina Frankham and Chris Caines

\section{Introduction}

Mobile screens have always been intimate displays. Attached to uniquely personal devices that rarely leave our sides and attached to networks that carry our most intimate communications, these screens carry with them a boutique set of opportunities and challenges for screen-based productions. In this chapter we examine these affordances and restrictions with particular focus on what sets these mobile screens apart from other screens as sites of reception and distribution. Particularly we focus on the materialities of these devices, the key aspects of the locative and the nature of mobile devices as networked screens. We also examine what it means that the mobile functions as both an extremely personal device while also acting as a site of consumption and distribution. As Farman posits (2016) the mobile can be considered not a medium as such but instead a locus that draws together a wide and diverse range of apps, practices and histories in one material networked form. In essence, mobile reception refers to a multi-stranded medium where elements can operate separately or combine into the hybrid forms that make the mobile a distinctive platform.

We draw on two key case study exemplars from mobile based media art productions that have made sophisticated use of these properties in ways that distinguish the mobile screen work as a modality with its own language, aesthetic rules and creative production strategies. Karen by the UK artist collective, Blast Theory (2015a) and The City of Forking Paths by the Canadian duo Cardiff and Miller (2014) display a nuanced sensitivity to the material circumstances of mobile screen production while achieving very different outcomes. In these examples we are looking at how media artists are continuing a tradition of experimentation with the materiality of new mediums and how this contributes to developing the language of the medium. We examine how the particular properties of the mobile screen influence the type of content that is produced. This examination ranges from the nature of the stories that sit well in the medium to the type of physical and virtual interaction that distinguish these screens from other networked screens.

\section{The specificity of mobile transmission}

Miniaturisation and the widespread availability of mobile data networks have enabled a broad diversity of what digital mobile media can be. As Farman notes 'nearly all digital devices fall 
under the category of "mobile", (Farman 2016, p. xii). In this chapter however, we are being very specific in focusing on mobile devices with screens that can deliver sound and moving image. In particular, through the examples we consider, the focus is on smartphones and pocket media players (such as iPods) as points where personal communication (as transmitted by messaging, video chat and other social apps) and media consumption (specifically of music, sound, still and moving images) converge.

Mobile as a delivery platform brings with it a wide range of technical variations that a maker must consider (Heyward 2014, pp. 121-133). Not just the divergence in operating systems, (Android, iOS, Windows Phone) but also differences in screen size, how the device fits into the user's hands, aspects of telecommunication infrastructure such as mobile network access, wireless network access as well as more localised interaction with other apps on the device such as alarms, calendars and notifications. The physicality of our connections to these devices and the ways we negotiate "familiar embodied and sensory" relationships with content on smartphones through touch (Pink et al. 2015, p. 238) demonstrate the uniqueness of smartphones when it comes to engaging with media. Drawing these particularities back up to the surface for consideration permits a more deliberate approach to practice that is critically and aesthetically active while simultaneously striving to offer content that is optimised for these exhibition circumstances.

Despite the seemingly intangible nature of moving image and sound, there are nonetheless, concrete, material circumstances connected with delivering content via a mobile device. This force of materiality on shaping content is reinforced by McLuhan's media theory, which Casemajor says highlights 'how the physical constraints of media shape the way information is produced' (2015, p. 5). Not only then is the McLuhan-esque dictum that 'the medium is the message' relevant to our examination of the mobile device but it also draws in the digital context of networks, software frameworks and device capabilities that comprise the specificities of this mode of screen content delivery.

An understanding of these material circumstances grants creators of mobile media opportunities to take advantage of particular affordances or find ways to accommodate device limitations. Characteristics such as being able to hold the device in your hand and move around while engaging with the screen are perhaps taken-for-granted features of viewing content on a smart phone. Yet these fundamental affordances have far reaching consequences 
for the kinds of content that can succeed in a mobile ecology that is growing in significance. The Deloitte Mobile Consumer Survey 2017 found that '[a]round 70 percent of 18-34 year olds watch videos on their smartphone weekly' (Drumm et al. 2017, p. 12). In this itinerant context, makers of non-site specific, mobile content need to think about where their users might be physically located (on a bus, in the bathroom, walking down the street) when experiencing the work. The cinema, lounge room or even the desktop computer screen are no longer the given as predominant venues for screen media. In addition, touch screens on mobile devices permit almost frictionless interaction with and between content elements $-\mathrm{a}$ feature which can be linked to a preference for media elements that are short and readily combined or linked with other similarly granular elements. It is a landscape where conventional rules for screen media production and how it is exhibited are being rethought and reimagined.

\section{Our intimate devices}

A common defining quality of locative and mobile media since it began to be recognized as a distinct form has been the effect of personal intimacy (Ito 2005) where a direct one on one relationship with the user/listener/viewer/reader is enabled. Works are often experienced solo, they whisper into your ear, relate personal stories and provide intimate experiences for you alone moving through that place at that time, or so they are designed to feel. A large part of this effect comes from the pre-existing, particularly private relationship we have with our mobile phones. Works that use mobiles for delivery and interaction in space are also freighted with the emotional bond we have with these most personal of devices and all for which they contain and serve as a conduit. The Sony Walkman is often cited as the progenitor to the intimacy effect present in mobile devices and media (Okada 2005). As a device for the delivery of personal music and sound, it sat at the intersection of the public and private, pioneering the experience of personal media combined with public space, generating in that intersection a personal cinema that included all you could see, generated by the juxtaposition of how you were choosing to soundtrack the spaces you were inhabiting and moving through.

\section{Audio and mobile screens}

Audio over mobile devices (especially mobile phones) carries all the historical associations and conventions of the personal phone call, freighted with the intimacy of a lifetime of oneon-one, direct voice to ear conversations. The reception experience of media works that use 
an intimate mode of address via audio as a central content strategy colours the way that the entire project is received by an audience, giving it the register of the personal and intimate through a mix of body memory and cultural association. This aspect of the mobile screen as both medium and delivery device, is made particularly evident in The City of Forking Paths, relying as it does (in a similar way to Karen) on a central figure playing the character of your guide, friend and co-adventurer throughout the work. Both works make conscious use of this particularity to heighten the bond between protagonist in each work and audience. In addition to this, both works rely on an intimate vocal performance that starts in the register of the familiar, the storytelling tone of an old friend and deepens from there into complex and affecting territory that implicate the viewer/listener into the webs of narrative that form the structure of these pieces. 


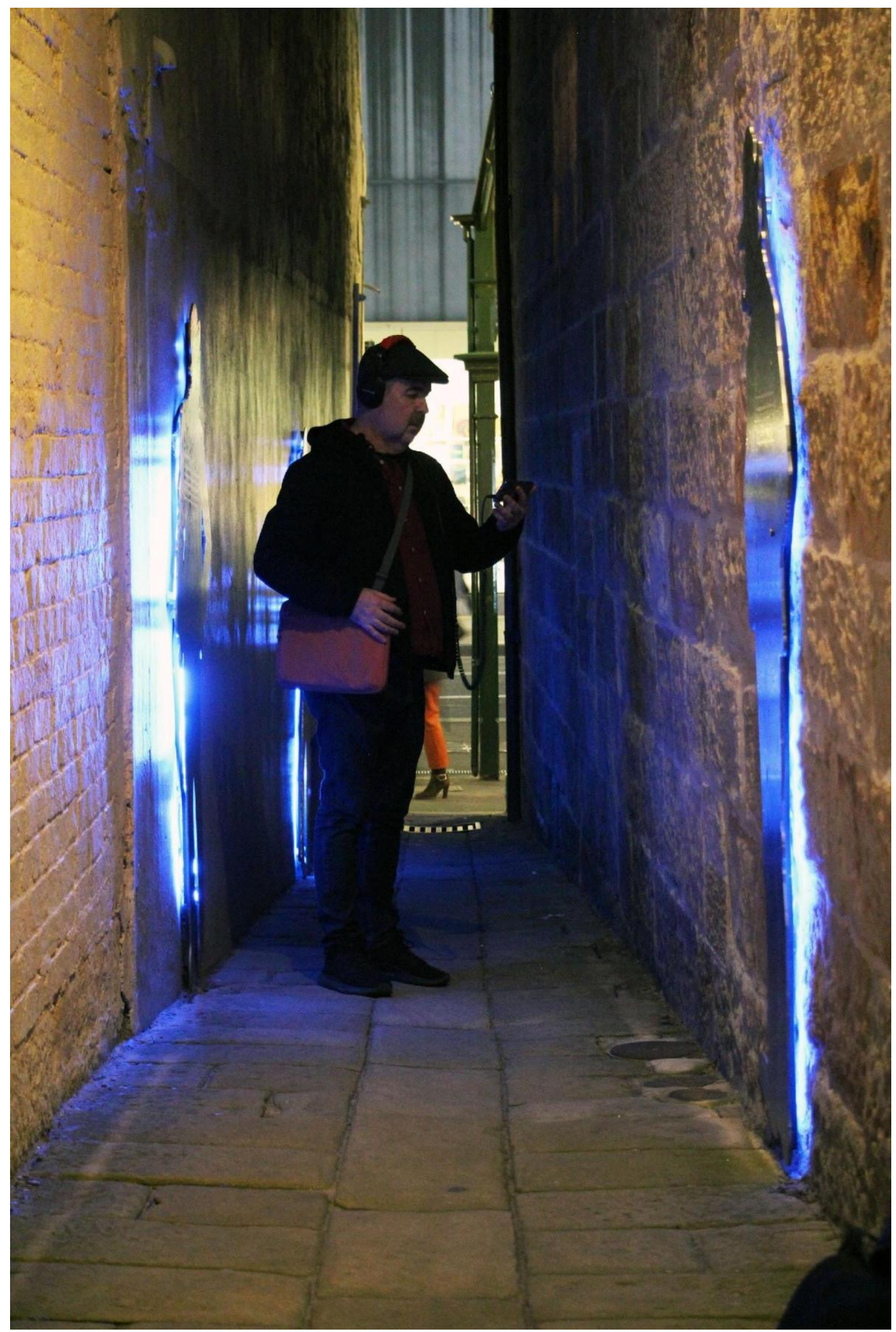

Figure 1: A user experiencing The City of Forking Paths via mobile phone in The Rocks, Sydney 


\section{The City of Forking Paths - Cardiff/Miller}

This site-specific work for The Rocks area of Sydney was commissioned as a permanent public artwork by the City of Sydney in 2014, and was featured in the Sydney Biennale of that year. Although primarily a guided video and binaural audio walk, it operates as an app on an iPod touch enabling it to be time locked and geo located. This means an audience cannot begin the work until after sunset and cannot start anywhere except Customs House in the centre of The Rocks area. Cardiff and Miller make use of a combination of immersive binaural soundscapes, personal voice driven narratives combined with a journey through specific locations to generate the collective ingredients that make up this work. Alongside these elements, The City of Forking Paths adds the effects of time to its palette.

Produced in early 2014, re-experiencing the work again at the time of writing (mid 2018) the user is struck by the contrast between then and now. That disjunction as an element of the piece creates a further level of imaginative engagement as the present and the past depicted in the work continue to diverge. To engage with the piece as time passes requires entering into the work increasingly as another world with temporal as well as spatial markers to navigate. For Cardiff, the element of binaural sound is key to, not only orientation in space, but also the physical immersion that she has developed as a way of heightening the tension between the imaginative space of the work being presented and the location in which it is presented.

I am interested in how audio affects our perception of the physical world. We understand three-dimensional space by using our vision, but also by the character of sounds we hear. If these sounds are manipulated and changed, then our perception of reality can be drastically affected. (Janet Cardiff's Audio Walks, 2011).

For Cardiff and Miller, working with intimate personal screens locatively develops from a longstanding practice with audio and specifically the audio walk as a genre. In her Walk Book (Cardiff \& Schaub 2005) Cardiff describes the combination of physical presence and the imaginative immersion that binaural audio makes possible as a key feature in audience engagement with a mobile work. The City of Forking Paths has been described (Barns 2014) as a type of 'physical cinema', which is achieved through the continuous immersion in spatial binaural sound throughout the work and also through the inclusion of physical locomotion as a core driving structure of the piece. 
Key to the locative video walking pieces produced by Cardiff and Miller is Cardiff herself as central narrator and guide. In The City of Forking Paths, as in many previous pieces going back as far as Forest Walk in 1991, Cardiff takes on a character that initially relaxes the listener/viewer, orienting them in a sort of guided mobile meditation. After this opening set up, the tenor of the piece subtly shifts, introducing narrative complexities and locative overlays that build an imaginative space between the real space inhabited by the audience and the screen space represented through the looking glass of the mobile device. This disjunction in situational awareness is exploited masterfully by Cardiff and Miller to create a hyper sensitivity to the conjunction of media and place in these works that, in a very real sense, is the central creative construct they are working with an audience member to build together.

In The City of Forking Paths Cardiff begins with the bored tone of an international artist commissioned to do yet another project in yet another foreign city. She listlessly describes what is around her, she complains that Miller is not with her; she regards the Rocks with no more engagement than a jaded tourist wandering the streets. Imperceptibly this tone changes, and before long you feel as if you are on an intense imaginative journey. The complexities of the piece reveal themselves and a viewer/listener begins to experience the work as a mix of reflective odyssey and locative play. Actors appear to play instruments and appear out of doorways, sounds come and go suggesting larger worlds outside this one and, in the hour the piece takes to experience before you return to Customs House, comes to an affecting resolution that could not be achieved without all the elements of sound, video, location and walking coming together to create a powerful mobile immersion.

\section{Karen app - Blast Theory}

Devised by UK artist group, Blast Theory, Karen is a smart phone application that uses the premise of interacting with an online life coach (the eponymous Karen, played by actress Claire Cage) as the basis for a choose-your-own-adventure-style, interactive drama. The work is described by Blast Theory as 'a hybrid, sitting somewhere between a game, a drama and a self-help quiz' (Adams 2015) and includes interactive elements such as sliding scale and multiple-choice personality profile questions. As part of the premise of engaging in life coaching sessions, the user receives mobile phone notifications from Karen when each episode can be accessed. Like The City of Forking Paths, content is time locked and is made available according to a pre-set schedule that shifts depending on when the previous 
instalment was completed. These appointments are also presaged by onscreen text at the end of each episode/session that alert the user to when the next session will be available.

Released first via the Apple App Store on 15 April 2015 and then a few months later for Android via the Google Play Store, comments left by users in the app stores indicate that the Karen app causes a level of genre confusion for some. For example:

\section{Not really very helpful. Too generic}

21 Jul 2017

\section{Mefightforthee}

This won't give you any information you can't get doing several of the less formal personality quizzes floating around. As a coach she is infuriatingly obsessed with telling you about her life, something that makes the process much MUCH longer and less professional than it should. If I was paying a life coach to help me and she spent this much time telling me about her love life I would fire her. We are here to work on my goals right? Furthermore the program promotes a very unprofessional attitude for a life coach who asks about her dress choice and personal problems. Who coaches in a terry robe?! My friend \& I also did it together and there is zero variation to Karen's actions or questions. No life coach would be so generically formulaic. As a psych student I don't think Karen is useful for anything beyond the superficial. If you have real issues go find a real person. (Apple 2017)

While it is solid advice to seek out a real person if you have real issues, the comment seems to indicate that the user has missed the playful and critical intentions of Blast Theory in creating the app. This reading that sits at odds with the maker intentions may be partially attributed to the infrastructure used to deliver the material to the audience. At over 5000 downloads of the Android version from the Google Play Store (2018) and over 10000 downloads for iOS in the first 4 months after its launch (Adams 2015), the piece achieves the kind of audience reach that has not been achieved with the other, more localised projects Blast Theory have undertaken. Consequently, there may be a high percentage of the audience for Karen who are engaging with the app without prior knowledge of Blast Theory and the kind of work for which they are known. For some users then, this lack of context finds its expression in frustration that the app does not perform in a way that is consistent with what 
some may perceive to be its genre, namely a personal growth or self-help tool rather than a theatrical (yet smart phone distributed) art work.

The choice to deliver the work via smartphone has undoubtedly enabled the user to experience additional levels of intimacy and personalisation and has taken the project into the private and personal spaces of participants' everyday lives. At the same time, however, the release via the App Store and Google Play means that Blast Theory has reached a very broad audience and has, by default, bought into the expectations that are associated with these distribution outlets. So, while the release of the Karen app has made it possible to produce something that takes full advantage of smartphone affordances, it could also be interpreted as a kind of platform trolling that only adds to the subversion and questioning central to the work. As Blast Theory self describe the purpose of the app:

We feel it's our job as artists to pose questions about this new world where technology is ever more personalised and intrusive. We love having our services tailored to us and we're scared of the price we're paying for that personalisation. (Blast Theory 2015b)

It is these tensions, between the desired effects and the consequences of their realisation, between the potentials of a platform and the genre expectations that come as part of the distribution strategy, that add depth and complexity to the already thought-provoking content presented in the Karen app.

\section{Mobile media as augmentation}

One of the characteristics of experiencing screen media on a mobile device can be an unexpected sense of immersion where the screen of the device ceases to be a barrier between viewer and content and transforms into a portal through which the two worlds (the user's world and the world-of-the-work) can connect. It is an unexpected transformation because immersion is usually associated with screens that occupy the full field of view and do so at a level of high audiovisual fidelity. In the case of pieces that use site specific spaces or locative techniques these spaces are already augmented by the audiences themselves. They are augmented by memory triggers and associations of place, augmented by the shifting environs the walker passes through, by the physical fact of inhabiting a moving gait with all the continual bodily information the very act produces as it happens. 
Of course, walking is also already a narrative, a chain of consecutive events in motion, a personal physical enaction of our seductive understanding of time as story. As has been noted by numerous walkers and thinkers we move through it as an act of bodily cogitation. As we walk and fall we join the dots, find the next foothold, sense the new direction. Any form of truly locative media (audio, text, image, video, AR) is designed to take these pre-existing augmentations as a given, as material to be used in the construction of overall affect. Locative media work is composed of a shifting collection of ingredients: site (the entire environment, static or in motion); physical intimate personal presence; relationship to and associations with media delivery mechanisms or devices and the media delivered in-situ. For the artist building work from these elements, the associations between the elements give rise to any gestalt impact the work has in the location. This can be thought of in much in the same way as the famous Kuleshov effect in cinematic editing. One of the building blocks of cinematic language, the Kuleshov effect notes the way an associative meaning is enabled through the links the viewer makes between individual shots.

In the case of Karen, a sense of co-presence and co-chronology is activated to achieve a feeling that the two worlds (of the user and of Karen) are coinciding with each other. Devices such as: direct address; Karen appearing to push buttons to bring up interactive question graphics; less than perfect framing (and at times handheld camera); spontaneous performance style; the sharing of personal details and spaces; and communication via SMS-style text messages all operate to conjure the illusion of a relational interaction so that the user feels they have some influence and agency in the exchanges.

In the experience of both The City of Forking Paths and Karen it can feel as if there is an added dimension that is accessed through the screen of the mobile that permits you to connect with a place that can feel no less real than the world we inhabit in our daily lives, in fact the screen and audio content overlays and interacts with our real world experience. With The City of Forking Paths, the screen acts as a portal between the two divergent versions of the locale - the one of our current experience and the enhanced version presented by Cardiff and Miller. In the case of Karen, the feeling can manifest as quite genuine concern for Karen's wellbeing, distaste for the boundary crossing into which the user is drawn or guilt when confronted by Karen over an intrusion into her bedroom. 
These effects are more than the kind of afterimages that Bunt (2013) describes as occurring when we process the content of an artwork or piece of media after encountering it in the gallery or cinema. The encounters described in relation to the examples in this chapter may be more correctly considered as augmentations because of the way the content intervenes and intrudes into the user's life. While narrative devices and emotional triggers are key to producing this sense of augmentation, the very nature of the device and the way it accompanies the user into all facets of their quotidian experiences is also significant in generating this effect.

The convergence between applications, practices and histories means that projects delivered via mobile screens can operate in ways that are akin to augmented and mixed reality where the sense of separation between the world of the participant and that of the content, and of the user existing outside the world of the work, is blurred. In these situations, the content adds, shapes and changes perceptions of the physical world of the user. Similar to effects described in relation to locative media earlier in this paper, there is potential to activate the affects, responses and motivations of the user to add narrative and emotional depth. These augmentations can be as a consequence, not just of specific content, but also because of the associations the user has with their device, as well as the affordances of the mobile device itself. This kind of emotional augmentation is very much the terrain in which both The City of Forking Paths and the Karen app are operating.

The Karen app intrudes on the personal world of the user by incorporating a variety of production techniques including narrative, performance style, camera technique, duration and scheduling alongside mobile specific affordances such as being able to send notifications, device portability and associations with intimate and/or personal communication. Backed by the central conceit of interactions with your life coach, Karen, the app gathers information about you, the user, through personality profiling questions alongside your responses to situations that are thrown up in the narrative that surrounds these interactions. This data about you then shapes the tone of your ongoing interactions and the tactics that Karen employs as your life coach.

However, the augmentation moves beyond the interjection of these personality-profiling questions to the user's everyday life. The scheduling of episodes, which constitutes the 
rhythm of the work, is irregular, and sessions can happen at odd times during the day from $8 \mathrm{am}$ through to $11 \mathrm{pm}$ at night.

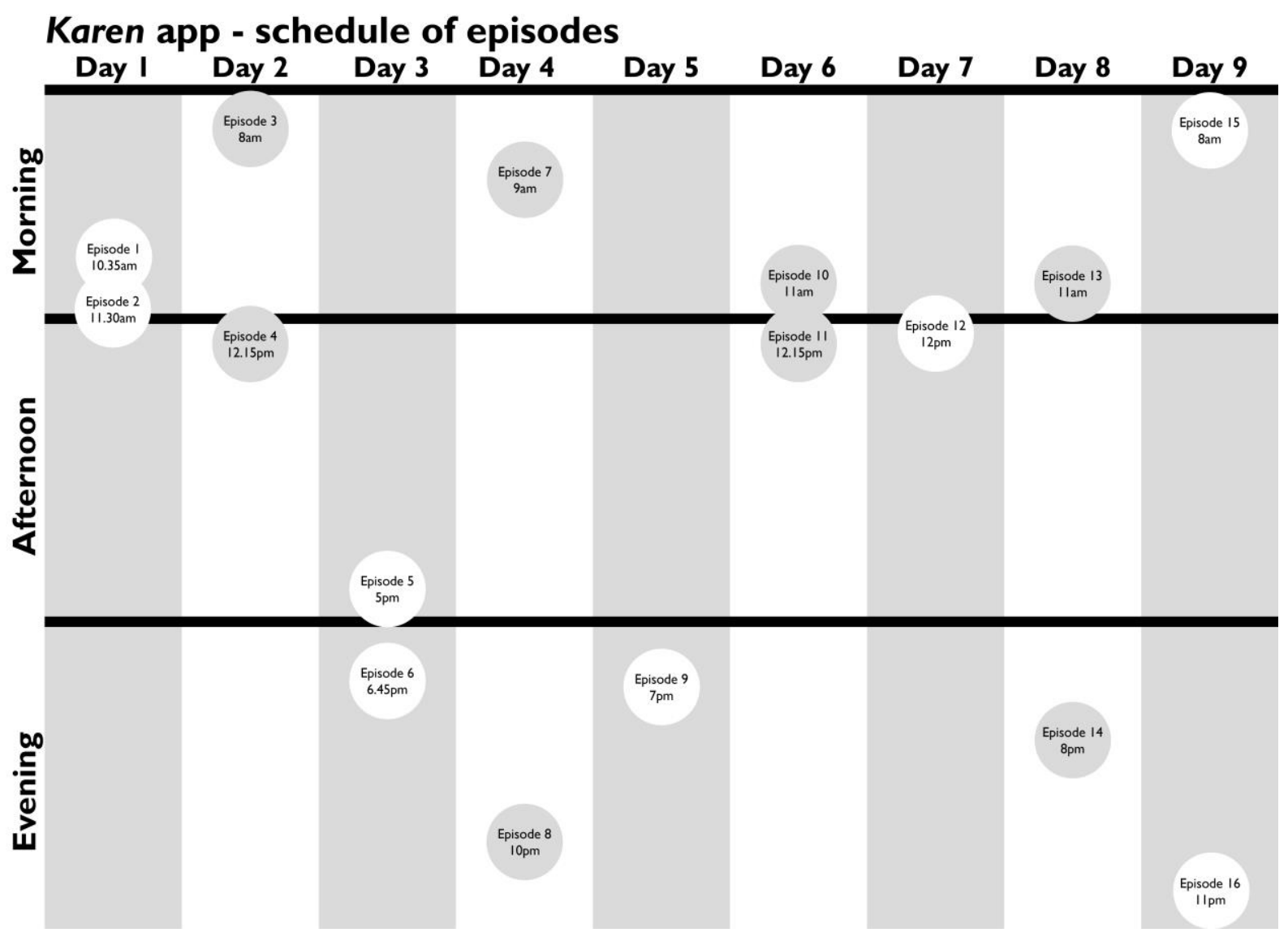

Figure 2: Schedule of Karen episodes showing spread of installments over 9 days

Even with the previously mentioned forewarning of when the next episode will occur, the messages from Karen/Karen can still trigger the kind of anticipation, delight or dread with which users would be familiar from other smartphone-based notifications such as text messages or social media interactions. There can also be multiple sessions occurring in a day, the content of which does not seem to be immediately relevant to the practice of life coaching (as evidenced by the previously cited user comment). But this is where the genius of the project lies. Yes, Karen seems a bit chaotic and perhaps 'a bit rubbish' as a life coach. However, as noted in the individually generated data report available to purchase upon completing the experience, '[p]erhaps it is through empathy with Karen that you reflect on your own capacities in your chosen area' (Blast Theory 2018).

These strategies aim to provoke reactions that can be added to the data on you as a subject and are 'used to personalise the experience' (Blast Theory 2018). The covert or embedded 
nature of these strategies, in that they are part of the drama that surrounds the life coaching sessions, means that they are potentially more likely to prompt authentic responses, evading the likelihood of respondents gaming the quizzes to present their most idealised self. There is an interesting effect that occurs whereby the user is simultaneously aware that answers will shift the dialogue with Karen in different directions but they also want to present an authentic version of themselves. As Mee observed, 'Although I was trying to "test" Karen's responses, and to see how our relationship would unfold if I answered her questions differently each time through, I almost always defaulted to truthful answers' (2016, p. 169). Mee's observation is backed up by Blast Theory's Matt Adams who said 'this was true of many participants, who found it more interesting to see how Karen/Karen responded to them [original emphasis] rather than to a fictitious version of themselves' (Mee 2016, p. 169).

Subjected to personal questions, contact at odd hours, disclosure about Karen's private life and a tone that is more co-conspirator than professional and client, the user is drawn into a kind of 'friend-crush' (Turney 2015) where they are destabilised from being outside of Karen's world and begin to have a stake in the course of her life. Indeed, Blast Theory are quite open about this manipulation, including outlining how user responses dictate the reappearance of certain motifs in later episodes 'as a reminder of your relationship with her [Karen]' (Blast Theory 2018).

The rhythm and frequency of interactions is not what one might expect of a life coach/client relationship, and this rhythm is integral to the kind of boundary blurring that Blast Theory is working to cultivate. The irregularity of the schedule fits with what Lustig describes as 'variable reward technology' (Brueck 2018) where the spikes of pleasure or reward are doled out by social media (and the internet more generally) at unpredictable times rather than consistently. The idea is an updating of Ferster and Skinner's general concepts of schedules of reinforcement, specifically the practice of a variable ratio schedule of reinforcement (1957) where 'the reinforcement occurs after a given number of responses, the number varying unpredictably from reinforcement to reinforcement (Ferster \& Skinner 1957, p. 391). Without the stability of a predictable, regular schedule of engagement, and given the vulnerable and at times volatile nature of Karen's character, the user can find themselves unsettled and emotionally engaged to a degree that is somewhat unexpected. 
Playing with the tropes of social media and the access to intimate modes that is enabled by mobile digital devices, these irregular intrusions generate a sense of co-presence that, as Berry describes in relation to the experience of social media more generally, can "transcend specific local time zones, seasons and weather so that people may be in a common social media space synchronously yet be experiencing completely different local physical conditions" (Berry 2017, p. 26). If the user responds immediately to the SMS style prompts about the availability of a new episode of Karen, then the time of day in Karen's location will match that of the user. However, if the user delays opening the app, the times of day slip out of synchronisation so that you may experience an interaction where it is late at night for Karen, but early in the morning for you. Nonetheless, the mode of direct, frequently intimate address and user familiarity with the sometimes aligned, sometimes unsynchronised patterns of consumption common to mobile media smooths over such disjunctions so that the exchanges seep into and overlay the user's everyday routine.

Augmentation is also enacted in the way that the user's personal histories are directly brought to bear on the shaping and structuring of the work. Indeed, there are times when the app relies on such interactions between user perceptions and the content as a means to personalise the experience and add complexity. The user's perceptual and emotional responses work with the sometimes blank, Kuleshov style performance techniques (where the actor's performance can be read in multiple ways depending on context) to provoke a deeper reading of what is going on under the surface of the exchanges with Karen and her flatmate Dave.

Karen regularly employs the classic tricks of the psychic and pop psychologist, making broad statements and summations that could apply to multiple situations but that are focused based on the data gathered by the app. In providing what can seem like wise insights ('I think you put off doing things that are difficult') the user's hunger for individual connection, which could be seen as a fundamental part of the contemporary human condition, is triggered. The user's state of mind lends the work emotional complexity and authenticity as they are directly implicated in the drama and denouement of the piece. Karen tells the user it is they who have precipitated her and Dave finally cutting the cords of their complicated relationship ('you set the cat among the pigeons' (Blast Theory 2015a)) and we get the impression that we are a direct catalyst for Karen moving on and moving out of the flat where most of the drama has taken place. 


\section{Conclusion}

With the increasing use of mobile devices as the screens of choice for consuming a variety of media content, there is considerable scope for screen producers to explore and experiment with the possibilities of mobile reception. In both The City of Forking Paths and Karen we see examples of mobile projects that make use of both the unique affordances and materiality of the mobile as context, setting and compositional material for the creation of new mobile screen-based works that exploit these qualities to great effect. These works offer distinctive examples of ways the Kuleshov effect can be applied to digital, interactive and mobile media in their combination of the inputs of place, media and viewer to create an experience that exceeds the content and boundaries of the small screen.

In The City of Forking Paths we see how Cardiff and Miller make use of both location and the act of walking in location in combination with the intimate performative voice and the displacement of binaural sound to create a powerful sense of mobile based immersion. As part of a trajectory of experimentation by the artists over 35 years, The City of Forking Paths pushes at the boundaries of content delivery and mobile media aesthetics to achieve a version of magic realism that is only possible through the combination of moving through The Rocks in Sydney guided by their video walk as augmentation. Their high-level mastery of the unique affordances and restrictions of mobile delivery enables a nuanced and affecting experience for the user that makes the most of the intimate status of the device and yet goes far beyond its usual quotidian and prosaic uses.

This intimate status is equally exploited by the Karen app as the user is drawn into a complicated sense of relationship with the central character of the piece at the same time as their personal data is harvested to direct the path of their ongoing in-app interactions. Interestingly, the very networks (i.e. the Google Play and Apple App stores) that facilitate this high degree of access to users' personal, digital spaces also produce a degree of genre confusion around the intention of the work. While this means that some users, who may be unfamiliar with the work of Blast Theory, do not get the playful and critical bent of the work, it can also be read as a kind of platform trolling that adds to the subversive purposes that underpin the Blast Theory oeuvre.

From our analysis of the creative choices, operation and reception of these two mobile media art projects we can see that a degree of augmentation is at play as the content overlays the 
users' real-world experiences with mobile media interventions. It is the interaction between user, device, content and location (in the case of The City of Forking Paths) that creates an unexpected sense of immersion that is less about the high fidelity of the media content and more a result of intense emotional engagement that is skilfully accreted over the course of each work. So, while technology is undoubtedly key in enabling these quality user media experiences, it is the confluence of media content, device associations and the interactions between components that set these works apart as thought-provoking exemplars of what is possible through the mobile reception of digital media art.

\section{References}

Adams, M. (2015). 'How we made experiential life-coaching app, Karen', Guardian (Culture professionals network), Fri 14 Aug 2015 21.40 AEST, viewed 20 May 2018 $<$ https://www.theguardian.com/culture-professionals-network/2015/aug/14/how-we-madelife-coaching-app-karen-blast-theory>.

Apple (2017). App Store Preview - Karen by Blast Theory, Ratings and Reviews, $<$ https://itunes.apple.com/au/app/karen-by-blast-theory/id945629374?mt=8>.

Barns, S. 2014, A wrinkle in time, a City of Forking Paths, viewed 20 September 2018 $<\mathrm{http}: / /$ sitesandsounds.net.au/forkingpaths/>.

Berry, M. (2017). Creating with Mobile Media, Palgrave Macmillan, USA.

Blast Theory (2015a). Karen, Google Play.

Blast Theory (2015b). Karen (Projects), viewed 10 May 20182018 $<$ https://www.blasttheory.co.uk/projects/karen/>.

Blast Theory (2018). 'Karen Data Report', personal communication, 31 August 2018.

Brueck, H. (2018). 'This is what your smartphone is doing to your brain -- and it isn't good', Business Insider Australia, 12 March 2018, viewed 10 May 2018 $<$ https://www.businessinsider.com.au/what-your-smartphone-is-doing-to-your-brain-and-itisnt-good-2018-3? $\mathrm{r}=\mathrm{US} \& \mathrm{IR}=\mathrm{T}>$. 
Bunt, B. (2013). Participation/Mediation, brogan bunt, vol. 2018, no January 16, 2013, viewed 20 June $2018<\mathrm{http}: / /$ www.broganbunt.net/?p=1949\%3E $>$.

Cardiff, J. and Miller, G.B. (2014). The City of Forking Paths, 19th Biennale of Sydney, Sydney.

Cardiff, J. and Schaub, M. (2005). Janet Cardiff: The Walk book, Thyssen-Bornemisza Art Contemporary (www.tba21.org/), Vienna.

Casemajor, N. (2015). Digital Materialisms: Frameworks for Digital Media Studies, Westminster Papers in Culture and Communication, vol. 10, no. 1, pp. 4-17.

Drumm, J., White, N., Swiegers, M. \& Davey, M. (2017). Smart everything, everywhere: Mobile Consumer Survey 2017 (The Australian cut), Deloitte Touche Tohmatsu Limited.

Farman, J. (ed.) (2016). Foundations of Mobile Media Studies: Essential Texts on the Formation of a Field, Routledge, Abingdon/New York.

Ferster, C.B. \& Skinner, B.F. (1957). Schedules of Reinforcement, Appleton-Century-Crofts, New York.

Google (2018). Play Store - Karen by Blast Theory, App store, $<$ https://play.google.com/store/apps/details?id=com.blasttheory.talktome $>$.

Heyward, M. (2014). Notes for walking an augmented landscape: Spatial narrative, walked practices and locative technologies. PhD thesis, University of Technology Sydney, Sydney, Australia.

Ito, M. (2005). Introduction, in M. Ito, D. Okabe \& M. Matsuda (eds), Personal, Portable, Pedestrian: Mobile Phones in Japanese Life, MIT Press, Cambridge, Mass., pp. 1-16. Janet Cardiff's Audio Walks. (2011). Fabrica: Brighton Centre for Contemporary Art, viewed 20 September $2018<$ https://fortynotes.wordpress.com/2011/03/03/janet-cardiffs-audiowalks/>. 
Mee, E.B. (2016). 'The audience is the message: Blast Theory's App-Drama Karen', TDR: The Drama Review, vol. 60, no. 3, pp. 165-171.

Okada, T. (2005). Youth culture and the shaping of Japanese Mobile Media: Personalization and the Ketai Internet as Multimedia, in M. Ito, D. Okabe \& M. Matsuda (eds), Personal, Portable, Pedestrian: Mobile Phones in Japanese Life, MIT Press, Cambridge, Mass., pp. 41 60.

Pink, S., Sinanan, J., Hjorth, L. \& Horst, H. (2015). Tactile digital ethnography: Researching mobile media through the hand, Mobile Media \& Communication, vol. 4, no. 2, pp. 237-251.

Turkle, S. (1995). Life on Screen: Identity in The Age of The Internet, Simon and Schuster, New York/ London/ Toronto/ Sydney.

Turney, E. (2015). App close and personal: Meet Karen, she wants your data, BBC, viewed 6 May 20182018

$<$ http://www.bbc.co.uk/programmes/articles/22HfmykfTdVfn2KZvFh7SBh/app-close-andpersonal-meet-karen-she-wants-your-data>. 